\title{
La receta médica: ¿reflejo condicionado o reflexión
} consciente?

Mucio Moreno, Oscar Arrieta, Rubén Burgos, Carlos Campillo, Miguel Á. Celis, Manuel de la Llata, Judith Domínguez, José Halabe, Sergio Islas, Luis Jasso, Alberto Lifshitz, Ricardo Plancarte, Alejandro Reyes-Sánchez, Guillermo Ruiz-Argüelles, Antonio Soda, Emma Verástegui y Julio Sotelo* Academia Nacional de Medicina de México, Comité de Ética y Transparencia en la Relación Médico-Industria, Ciudad de México, México

\section{Resumen}

Extender una receta o indicar un tratamiento suele ser la última parte de la consulta médica. Este proceso crucial puede desvirtuarse debido a múltiples factores como capacidad prescriptiva limitada, exceso de trabajo y falta de reflexión o tiempo. La información insuficiente acerca del paciente o del tratamiento afecta el proceso prescriptivo y propicia errores que pueden ser graves para la salud del enfermo. La Academia Nacional de Medicina, en consonancia con la Organización Mundial de la Salud, hace énfasis en hacer del proceso prescriptivo un ejercicio de reflexión.

PALABRAS CLAVE: Prescripción médica. Receta médica. Medicamentos. Diagnóstico médico. Tratamiento médico. Farmacoterapia.

\section{Medical prescription: conditioned reflex or conscious reflection?}

\begin{abstract}
Writing a prescription or indicating a treatment is usually the last part of medical consultation. This crucial process can be undermined by multiple factors such as limited prescriptive ability, overwork, and lack of reflection or time. Insufficient information about the patient or the treatment affects the prescriptive process and leads to errors that can be serious for patient health. The National Academy of Medicine, in line with the World Health Organization, emphasizes the relevance of making the prescriptive process a reflective exercise.
\end{abstract}

KEY WORDS: Medical prescription. Medicines. Medical diagnosis. Medical treatment. Pharmacotherapy.

Elaborar la receta para el paciente suele ser la última parte de la consulta médica. Este acto aglutina el esfuerzo del ejercicio diagnóstico, cristaliza la agudeza del criterio profesional y le da congruencia a la ciencia médica. Es muy relevante que la prescripción médica incluya en toda ocasión las cuatro "erres" (en un ejercicio nemotécnico): debe ser razonable, razonada, racionada y racionalizada. Es igualmente importante insistir en la caligrafía del médico en la prescripción, que siempre debe ser clara y entendible.

Cada vez con mayor frecuencia el paciente se involucra activamente en lo que el médico le prescribe, está mejor informado sobre su padecimiento, opciones de tratamiento, riesgos y complicaciones; con
Correspondencia:

*Julio Sotelo

E-mail: jsotelo@unam.mx

0016-3813/৫ 2020 Academia Nacional de Medicina de México, A.C. Publicado por Permanyer. Este es un artículo open access bajo la licencia CC BY-NC-ND (http://creativecommons.org/licenses/by-nc-nd/4.0/). 
todo ello se eleva su exigencia hacia el médico, con la expectativa de participar en la elección prescriptiva.

Los malos hábitos de prescripción son origen de tratamientos inefectivos e inseguros que pueden exacerbar o alargar la enfermedad, producir tensión y daño al paciente y costos más altos, como advierte la Organización Mundial de la Salud (OMS). ${ }^{1}$ Al analizar la capacidad prescriptiva de médicos jóvenes recién egresados que fueron evaluados en pregrado y seis meses después de su graduación, se observó que la mitad seleccionó el medicamento inadecuado o dudoso, un tercio no escribió correctamente la receta y dos tercios no pudieron sustentar académicamente el medicamento prescrito. Algunos médicos señalaban que sus hábitos de prescripción mejorarían después al terminar sus estudios de pregrado, sin embargo, la investigación demostró que las aptitudes prescriptivas no tuvieron grandes avances después de la graduación a pesar de adquirir experiencia en general.

Durante la formación clínica no se enfatiza suficiente sobre cómo elegir un fármaco y la información que debería acompañar el acto prescriptivo. La mayoría de las escuelas de medicina se enfocan principalmente en el conocimiento farmacológico teórico e invierten poco esfuerzo en la adquisición de capacidades prescriptivas.

La OMS advierte que los malos hábitos de prescripción contribuyen a que el prescriptor sea vulnerable a influencias que pueden promover una prescripción irracional, como la presión del paciente, las malas prácticas de colegas y la información de los representantes de los laboratorios. Prescribir debe formar parte de un proceso lógico deductivo, basado en información global y objetiva. No debe ser un acto reflejo o una respuesta a presiones comerciales.

Con la experiencia de un estudio en estudiantes de medicina enfocado al proceso de prescripción, la OMS propuso una "guía de la buena prescripción", la cual fue revisada por expertos en enseñanza de farmacoterapia. Independientemente del nivel de experiencia profesional, es recomendable revisar si en nuestra práctica médica cumplimos con el proceso de terapéutica razonada recomendada por la OMS en seis pasos, los cuales resumimos a continuación:

1. Definir el problema del paciente.

2. Especificar el objetivo terapéutico, ¿qué se desea conseguir con el tratamiento?

3. Comprobar si el tratamiento es adecuado, efectivo y seguro.
4. Iniciar el tratamiento.

5. Brindar información, instrucciones y advertencias.

6. Supervisar (¿y suspender?) el tratamiento.

En la práctica, cuando un médico atiende diariamente de 30 a 40 pacientes en una institución es difícil que pueda cumplimentar un proceso terapéutico razonado en cada caso. Considerando que no siempre será posible seguir paso a paso las recomendaciones de la OMS para la "buena prescripción", en los siguientes párrafos el Comité de Ética y Transparencia en la Relación Médico-Industria (CETREMI) ${ }^{2}$ sugiere las siguientes consideraciones en torno a estas seis propuestas:

- Mediante un análisis cuidadoso, historia clínica estructurada y examen físico completo se podrá definir con claridad el padecimiento y la ansiedad asociada, que pueden requerir de ayuda adicional, así como la dificultad en la adhesión al tratamiento. El éxito de la intervención médica dependerá de la selección adecuada de la prescripción. En muchos casos no es necesaria la prescripción de fármacos. La presión del paciente para recibir algún medicamento o tratamiento específico puede enmascarar un problema adicional.

- La definición clara del objetivo terapéutico es esencial para una adecuada prescripción y evitará el uso de fármacos innecesarios, como prescribir antibióticos o antiparasitarios en caso de fiebre, o una pomada con corticoides y antibióticos por no diferenciar entre una micosis y un eccema, etcétera. Siempre es buena idea discutir el objetivo terapéutico con el paciente, ya que lo convierte en "socio" informado y mejora la adhesión terapéutica. El arte de la prescripción médica consiste en diferenciar a cada paciente como un caso particular del resto.

- La principal característica para indicar un tratamiento o medicamento es su eficacia, sin embargo, también deberá ser seguro y conveniente. Por diversos mecanismos, muchos de ellos genéticos, un paciente puede ser diferente al promedio, con diferencias en la farmacodinamia y farmacocinética del medicamento que modifican el margen y la ventana terapéutica. Clínicamente esto pudiera generar "resistencia" o "hipersensibilidad" al medicamento.

- La pauta de dosificación debe ser conveniente, es decir, entre más complejo es el esquema de dosificación, menos conveniente. Se logra que un fármaco alcance su concentración ideal en sangre cuando existe un equilibrio entre su absorción, distribución, metabolización y excreción. 
Cualquier modificación de estos factores influye sobre su potencial eficacia y aunque se puede medir el nivel plasmático de un medicamento, hacerlo es costoso y difícil de interpretar; es más sencillo buscar signos clínicos de respuesta y de efectos secundarios. La duración del tratamiento y la dosis de los fármacos también deben ser individualizadas. Por ejemplo, muchos pacientes ambulatorios con cáncer padecen dolor y el médico teme prescribir morfina por periodos prolongados, confundiendo tolerancia con adicción.

- La adhesión del paciente a tratamientos prolongados resulta problemática. Frecuentemente, el paciente suspende un fármaco cuando los síntomas mejoran o aparecen efectos indeseados. Por comodidad del paciente y del médico es frecuente la "renovación automática" de la receta; sin embargo, esta es una razón de prescripción excesiva o innecesaria, particularmente en pacientes con enfermedades crónicas. Es importante enfatizar que el médico es responsable de que la receta sea clara y legible, indicando con precisión los datos que identifiquen al paciente (nombre, dirección y edad), la fecha, nombre genérico del medicamento, cantidad total y dosis exacta, frecuencia y vía de administración, periodo del tratamiento, instrucciones y advertencias, firma del médico responsable e información de contacto.

- La estadística indica que la mitad de los pacientes no toma el medicamento correctamente. En algunos estudios menos de $60 \%$ de los pacientes entienden cómo deben tomar el medicamento. Cada paciente requiere comprender las instrucciones del médico y resolver dudas o inquietudes. Tener demasiados pacientes no debe ser excusa para evitar instruirlos adecuadamente respecto a su prescripción médica. ${ }^{3}$
La supervisión del tratamiento permite al médico determinar si ha obtenido el resultado esperado. Para lograrlo, será necesario mantener comunicación adecuada con el paciente.

El médico está obligado a conocer los avances farmacológicos. La información comercial que comúnmente manejan las empresas farmacéuticas en campañas promocionales puede ser tendenciosa y no sustentada académicamente.

\section{Conflicto de intereses}

Los autores declaran no tener conflicto de intereses alguno.

\section{Financiamiento}

Los autores no recibieron patrocinio para llevar a cabo este artículo.

\section{Responsabilidades éticas}

Protección de personas y animales. Los autores declaran que para esta investigación no realizaron experimentos en seres humanos ni en animales.

Confidencialidad de los datos. Los autores declaran que en este artículo no aparecen datos de pacientes.

Derecho a la privacidad y consentimiento informado. Los autores declaran que en este artículo no aparecen datos de pacientes.

\section{Bibliografía}

1. Tichelaar J, Richir MC, Garner S, Hogerzeil H, de Vries PGM. WHO guide to good prescribing is 25 years old: quo vadis? Eur J Clin Pharmacol. 2020;76:507-513

2. Academia Nacional de Medicina. Comité de Ética y Transparencia de los Médicos con la Industria Farmacéutica (CETREMI). Gac Med Mex. 2015;151:293.

3. Celis MA, Halabe J, Arrieta A, Burgos R, Campillo C, de la Llata M, et al. El consentimiento informado: recomendaciones para su documentación. Gac Med Mex. 2019;154:716-718. 